\title{
DEVELOPMENT OF AN INVERSE ESTIMATION METHOD OF SEA SURFACE DRAG COEFFICIENT UNDER STRONG WIND CONDITIONS
}

\begin{abstract}
Masaki Yokota ${ }^{1}$, Noriaki Hashimoto ${ }^{1}$, Koji Kawaguchi ${ }^{2}$, and Hiroyasu Kawai ${ }^{2}$
For the purpose of clarifying the mechanism of energy transfer from winds to waves under strong wind condition, the ADWAM, a wave prediction model incorporating the data assimilation method, was modified to deduce the sea surface drag coefficients as its control variables. Then, validity of the model was verified through the identical twin experiment in deep sea conditions. Also, the behavior of the deduced parameter was examined through several experiments. As a result, it was confirmed that the drag coefficient deduced by the model is accurate enough when the number of the given observation data is sufficient compared with the number of the unknown parameters. It was also confirmed that the accuracy of the deduced coefficient can be improved by adding an a priori condition even if the number of the observation data is insufficient.
\end{abstract}

Keywords: sea surface drag coefficient; data assimilation; adjoint WAM

\section{INTRODUCTION}

Recently, the third generation wave models, particularly WAM, SWAN and Wave Watch III, are widely used in many countries. These models can certainly describe wave status more accurately than the previous models, and have been applied to various practical applications such as clarification of causes of disasters due to waves and interpolation of missing wave observation data, etc. However, when we apply these models to actual data especially in severe storm conditions, we still need to tune some parameters in the models by trial and error manner to adjust the overestimated or underestimated wave height comparing with the observed one.

In the wave prediction models, the sea surface drag coefficient $C_{D}\left(=U_{*}^{2} / U_{10}^{2}\right)$, the important factor of the energy transfer process from winds to waves, is generally approximated to a linear function of wind speed proposed by Wu (1980) or Mitsuyasu \& Honda (1982) as shown in Fig 1 by Eqs. (1) and (2) respectively.

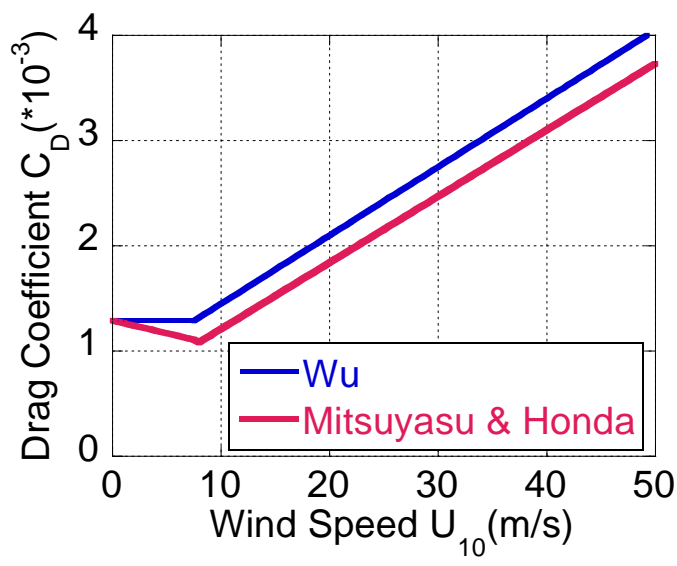

Figure 1. Drag coefficients proposed by Wu (1980) and Mitsuyasu et.al. (1982)

$$
\begin{gathered}
C_{D}=1.2875 \times 10^{-3} \quad U_{10} \leq 7.5 \mathrm{~m} / \mathrm{s} \\
C_{D}=\left(0.8+0.065 \times U_{10}\right) \times 10^{-3} \quad U_{10} \geq 7.5 \mathrm{~m} / \mathrm{s} \\
C_{D}=\left(1-1.89 \times U_{10} \times 10^{-2}\right) \times 1.28 \times 10^{-3} \quad U_{10} \leq 8 \mathrm{~m} / \mathrm{s} \\
C_{D}=\left(1+1.078 \times U_{10} \times 10^{-1}\right) \times 5.81 \times 10^{-4} \quad U_{10} \geq 8 \mathrm{~m} / \mathrm{s}
\end{gathered}
$$

\footnotetext{
${ }^{1}$ Kyushu University, 744 Motooka, Nishi-ku, Fukuoka, 812-8581, Japan

${ }^{2}$ Port and Airport Research Institute, 3-1-1, Nagase, Yokosuka, 239-0826, Japan
} 
However, these empirical equations were deduced from the observational and the experimental data under wind speed conditions slower than $25 \mathrm{~m} / \mathrm{s}$ at the fastest and were extrapolated to faster wind speed conditions. Accordingly, accuracy of the computed wave height under strong wind conditions that cause severe disaster seems to be unreliable. Actually, the recent report of an aerodynamic observation suggested that the sea surface drag coefficient declines when wind speed exceeds about 30m/s (Powell et. al., 2003). This result seems to be very important for forecasting the disaster caused by an intense typhoon under the global warming.

To verify this fact, wave observations right under a severe typhoon is quite difficult and not realistic due to low feasibility. The preparation of the experimental equipment for the measurement is also very expensive. Theoretical approach for clarifying the mechanism of the energy transfer under strong wind condition is difficult because the mechanism includes very complicated physical processes such as wave breaking and spray. As an alternative method the inverse estimation method that presume internal structure through the model that utilizes the past wave observation data is considered as applicable. In this study, a data assimilation method was introduced into WAM to deduce a realistic and appropriate drag coefficient, and the validity of the method was examined by several wind and wave conditions.

\section{INVERSE ESTIMATION METHOD}

Sea Surface Drag Coefficient Assumed as a Piecewise-constant Function

First of all, the expression of the energy transfer term in WAM Cycle4, $S_{\text {in }}$, was modified by replacing Janssen's theory with Mitsuyasu \& Honda's equation (1982) that use sea surface drag coefficient. After that, in order to deduce the sea surface drag coefficient $C_{\mathrm{D}}$ as an arbitrary function with respect to wind speed, it was the sea surface drag coefficient was assumed as a piecewise-constant function over the wide wind speed range as shown in Fig 2.

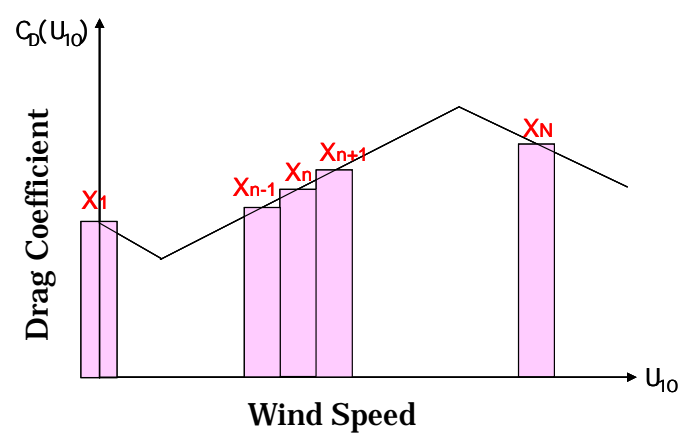

Figure 2. Drag coefficient assumed as a piecewise-constant function

\section{Data Assimilation Method}

The adjoint method, one of the data assimilation methods, used in this study is considered to be a tool of remote sensing with respect to not only space but also time in the past and at present, i.e., that utilizes observation data to connect the model with the reality when we try to reproduce natural phenomena by a model. The adjoint method can presume the optimum value of the unknown parameter including nonlinearity by using the maximum likelihood estimation method. Hersbach (1998) introduced the adjoint method into WAM and developed the adjoint model ADWAM. The adjoint model gives a better prediction by correcting several model parameters in the WAM. In the adjoint model, the most suitable parameters are automatically estimated by minimizing the cost function composed of the error margin of estimation and observed values, expressed by Eq. (3).

$$
J(x)=\sum_{t=0}^{\mathrm{T}} \frac{1}{2}\left(H_{t}(x)-y_{t}\right)^{T} \mathbf{R}_{t}^{-1}\left(H_{t}(x)-y_{t}\right)
$$

where $x$ is the vector of the model parameters, and $y_{t}$ is the vectors of observed values, and $H_{t}$ is the matrix of the operator converting the model state $x$ into $y_{t} . \mathbf{R}_{t}$ is the covariance matrix of the observation errors, and is expressed as follow: 


$$
\mathbf{R}_{t}=<\Delta y_{t}\left(\Delta y_{t}\right)^{\mathrm{t}}>
$$

where $<>$ denotes the ensemble average, and $\Delta y$ is the vector of the observation errors.

The minimization of the cost function is necessary to obtain the optimum value of $x$ but analytical approach for the minimization is not easy. Instead, a method of descent is usually applied, which requires the following descendent value of the cost function $(g=\partial J / \partial x)$.

$$
g=\mathrm{H}_{t}^{T}(x) \mathbf{R}_{t}^{-1} \sum_{t=0}^{\mathrm{T}}\left(H_{t}(x)-y_{t}\right)
$$

The transpose of the operator matrix, $\mathrm{H}^{T}$, is necessary to estimate the descendent value $g$. This matrix corresponds to the adjoint operator $\mathrm{H}^{*}$ of the tangent liner operator of $\mathrm{H}$.

The computation of Eq. (5), it is directly computed through the adjoint run with the adjoint model code. We made the adjoint code of WAM using AMC (Adjoint Model Compiler, Giering, 1995). A descent method with Quasi-Newton Method was used for the minimization of Eq. (3).

The procedure of data assimilation with adjoint WAM is almost the same as that of Hersbach (1998). In normal applications of wave hindcasting, the WAM code is used and the energy balance equation is integrated in the forward direction of time $t$ (forward run). On the other hand in data assimilation, the ADWAM code is used and the equation is integrated in the reverse direction of time $t$ (adjoint run) to obtain the information with respect to the control parameters to be modified for data assimilation.

If nonlinearity is included in the forward run, the nonlinearity should be stored at each integration time step. To avoid the storage problem in the computations for realistic applications, a 'check-point' method has been implemented in the model the same as WAM Cycle 5 (Hersbach, 1998).

\section{A Priori Condition}

In the field of atmospheric research, data assimilation has generally been used for the correction of the initial state in the forecast, where the approximate value was predictable beforehand. This necessitates the introduction of the restriction condition that the optimum control parameters should exist in the vicinity of the initial guess of the computation. In the general form, the cost function is defined as the sum of the background error term and observation error term as expressed by Eq. (6).

$$
\begin{aligned}
J(x)= & \frac{1}{2}\left(x-x_{o}\right)^{T} \mathrm{~B}_{t}^{-1}\left(x-x_{o}\right) \\
& +\sum_{t=0}^{T} \frac{1}{2}\left(H_{t}(x)-y_{t}\right)^{T} \mathbf{R}_{t}^{-1}\left(H_{t}(x)-y_{t}\right)
\end{aligned}
$$

where $x_{o}$ is the background model parameters (e.g. the first guess), and $\mathrm{B}_{t}$ is the covariance matrix of the background errors.

In this new adjoint WAM, the background error term that restricts the initial condition is not necessary because the optimum parameters in high wind speed are uncertain at present. However, since the division number of the wind speed in the assumed piecewise-constant function is equal to the number of unknown parameters in the model, the larger the assumed division number is, the more difficult and unstable the inverse estimation of parameters become. This is namely an ill-conditional inverse problem and the minimization of the cost function composed of only the observation errors leads to unstable and unreliable computation. To solve this problem, two types of a priori conditions, that the sea surface drag coefficient is continuous and smooth between the adjoining wind speeds was added as a background error term to the cost function in the adjoint model of WAM , as expressed by Eqs. (7) and (8).

$$
\begin{aligned}
J(x)= & W \sum_{n=1}^{\mathrm{N}}\left(x_{n}-x_{n-1}\right)^{T} \mathrm{~B}_{t}^{-1}\left(x_{n}-x_{n-1}\right) \\
& +\sum_{t=0}^{\mathrm{T}}\left(H_{t}(x)-y_{t}\right)^{T} \mathbf{R}_{t}^{-1}\left(H_{t}(x)-y_{t}\right)
\end{aligned}
$$




$$
\begin{aligned}
J(x)= & W \sum_{n=1}^{\mathrm{N}}\left(x_{n+1}-2 x_{n}+x_{n-1}\right)^{T} \mathrm{~B}_{t}^{-1}\left(x_{n+1}-2 x_{n}+x_{n-1}\right) \\
& +\sum_{t=0}^{\mathrm{T}}\left(H_{t}(x)-y_{t}\right)^{T} \mathbf{R}_{t}^{-1}\left(H_{t}(x)-y_{t}\right)
\end{aligned}
$$

The most reasonable and suitable parameters are inversely estimated by minimizing the cost function assumed as the summation of the observation error (the difference between the observed wave data and the hindcasted wave data) and the background error (the degree of the satisfaction of a priori condition). In addition, a weighting coefficient $W$ is introduced between the observation error and the background error and the dependence property of $W$ in the inverse estimation is also discussed by changing the weighting coefficient $W$ in the computations.

\section{VERIFICATION OF THE NEW METHOD}

\section{Identical Twin Experiment}

The validity of the new inversion method was verified unknown drag coefficients being corrected to the vicinity of the target value from an arbitrary initial value by assimilating the time series of the computed wave height. Fig 3 shows the flow of the identical twin experiment. First, the target drag coefficient was assumed as a function of wind speed. This study assumed eq.(9) as a target value, which declines in wind speed higher than $30 \mathrm{~m} / \mathrm{s}$.

$$
\begin{array}{ll}
C_{D}=\left(1-1.89 \times U_{10} \times 10^{-2}\right) \times 1.28 \times 10^{-3} & U_{10} \leq 8 \mathrm{~m} / \mathrm{s} \\
C_{D}=\left(1+1.078 \times U_{10} \times 10^{-1}\right) \times 5.81 \times 10^{-4} & 30 \mathrm{~m} / \mathrm{s} \geq U_{10} \geq 8 \mathrm{~m} / \mathrm{s} \\
C_{D}=\left(7.5-1.078 \times U_{10} \times 10^{-1}\right) \times 5.81 \times 10^{-4} & 50 \mathrm{~m} / \mathrm{s} \geq U_{10} \geq 30 \mathrm{~m} / \mathrm{s}
\end{array}
$$

Then, the time series of the wave height at the center of a sea area (in Fig. 5) was computed with the target function. These time series data were used as the wave observation data in the numerical experiments. Next, the unknown function was inversely estimated from an arbitrary initial value by assimilating the wave observation data. Finally, the estimated functions were compared with the target functions.

Fig. 4 compares the time series of the computed wave height with that of the target value (solid line), the initial parameter (broken line) and the deduced parameter (white circles). Although the wave height computed with the initial parameter of Mitsuyasu \& Honda (1982) overestimate the peak of the wave height, the wave height computed with the inversely deduced parameter shows good agreement with the observed (target) data.

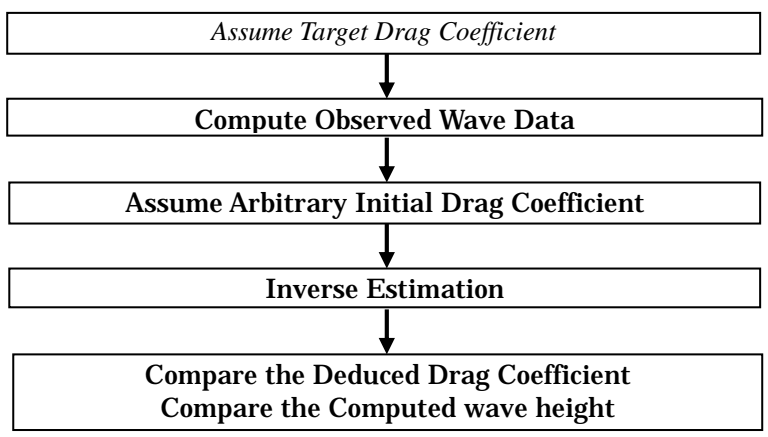

Figure 3. Flow of identical twin experiment 


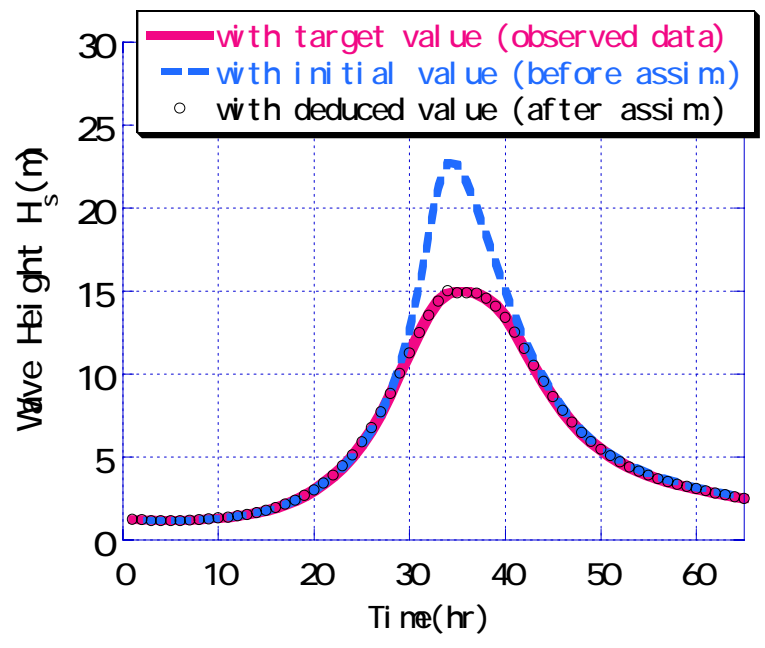

Figure 4. Comparison of the time series data (the initial values (the broken line), the target values (the solid line), and the deduced values (the white circles))

\section{Simulation Conditions}

The condition of the numerical simulation is that a typhoon passes through a rectangular sea area of the computation area from the south toward the north. The dimension of the computational area is 10 degrees in latitude by 10 degrees in longitude, and the grid interval is 0.5 degree. The sea surface wind was estimated by an empirical parametric typhoon model. To focus on intense wind condition higher than $30 \mathrm{~m} / \mathrm{s}$, we assumed the central atmospheric pressure $850 \mathrm{hPa}$, the maximum wind speed radius $100 \mathrm{~km}$ and migration velocity $50 \mathrm{~km} / \mathrm{h}$. Fig. 5 shows an example of the wind field $(\mathrm{t}=24 \mathrm{~h})$ and the time series of the wind speed at the center of the computational area. As seen in the figure, the maximum wind speed exceeds $40 \mathrm{~m} / \mathrm{s}$.
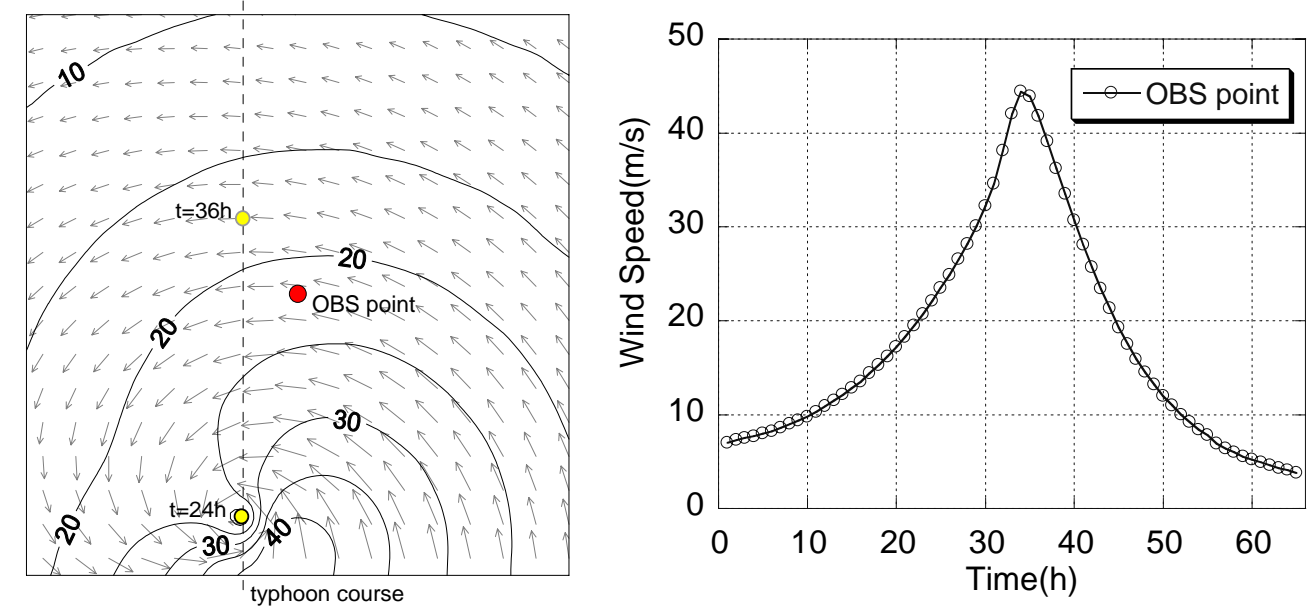

Figure 5. Example of wind field and time series of wind speed

\section{Characteristics of Estimated Parameter}

The behavior of the estimated drag coefficient was variously investigated by changing a) the number of unknown parameters, b) the number of observation data, c) the type of a priori conditions and the weighting coefficient, and d) the type of initial value and the weighting coefficient. The examination cases are shown in Table 1. 
Table -1 Examination cases

\begin{tabular}{|c|c|}
\hline \multirow{3}{*}{$\begin{array}{l}\text { a) compare the difference of the number of unknown } \\
\text { parameter without a priori condition } \\
\text { ( the number of observation data : } 65 \text {, } \\
\text { initial value of parameter : Mitsuyasu \& Honda ) }\end{array}$} & $100(0.5 \mathrm{~m} / \mathrm{s}$ interval $)$ \\
\hline & $50(1 \mathrm{~m} / \mathrm{s}$ interval $)$ \\
\hline & $25(2 \mathrm{~m} / \mathrm{s}$ interval $)$ \\
\hline \multirow{3}{*}{$\begin{array}{l}\text { b) compare the difference of the number of observation } \\
\text { data without a priori condition } \\
\text { ( the number of unknown parameter : } 25 \text {, } \\
\text { initial value of parameter : Mitsuyasu \& Honda ) }\end{array}$} & 65 ( $1 \mathrm{~h}$ interval ) \\
\hline & 33 ( $2 \mathrm{~h}$ interval ) \\
\hline & 11 ( 6h interval ) \\
\hline \multirow{4}{*}{$\begin{array}{l}\text { c) compare the difference of weighting coefficient with } \\
\text { two type of a priori condition } \\
\text { ( the number of unknown parameter : } 100, \\
\text { the number of observation data }: 65 \text {, } \\
\text { initial value of parameter : Mitsuyasu \& Honda ) }\end{array}$} & $W=0$ \\
\hline & $W=10^{3}$ \\
\hline & $W=10^{4}$ \\
\hline & $W=10^{5}$ \\
\hline \multirow{3}{*}{$\begin{array}{l}\text { d) compare the difference of weighting coefficient with } \\
\text { of a priori condition } \\
\text { ( the number of unknown parameter : } 100 \text {, } \\
\text { the number of observation data : } 65 \text {, } \\
\text { initial value of parameter : constant by } 1 * 10^{-3} \text { ) }\end{array}$} & $W=0$ \\
\hline & $W=10^{3}$ \\
\hline & $W=10^{4}$ \\
\hline
\end{tabular}

Figure 6 compares the deduced value with the target value where the computations are carried out for different number of unknown parameter $(=100,50,25)$ without adding a priori condition. The figure shows that the value in the wind speed of higher than $30 \mathrm{~m} / \mathrm{s}$ (where the initial value was intentionally separated from the target value) was corrected to the vicinity of the target value from the initial value. However, the deduced value is unstable for large number of the unknown parameter $(=50$, 100) while the drag coefficient deduced by the inversion method is accurate if the number of observation data $(=65)$ is sufficient compared with the number of unknown parameter $(=25)$. Because the maximum wind speed generated in this typhoon is approximately $45 \mathrm{~m} / \mathrm{s}$, the value in the wind speed higher than $45 \mathrm{~m} / \mathrm{s}$ is not corrected from the initial value.
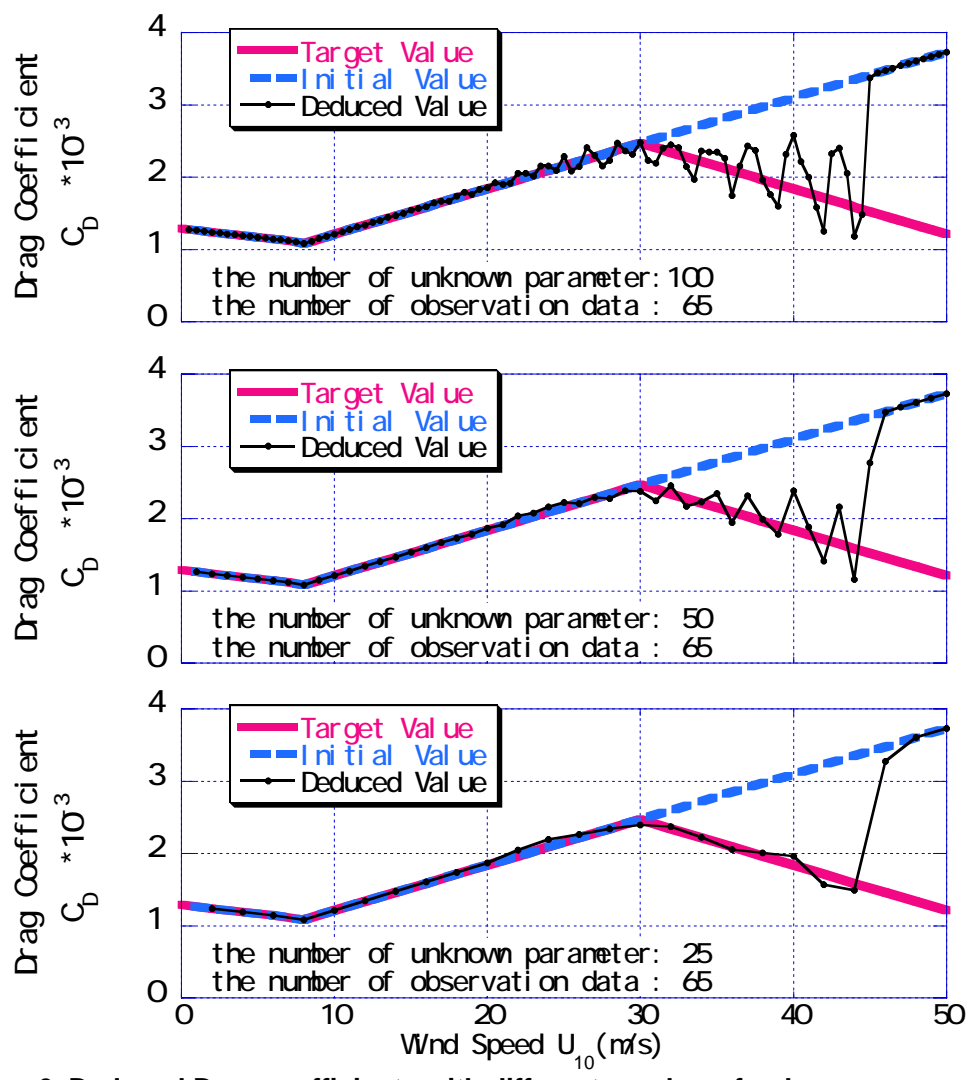

Figure 6. Deduced Drag coefficients with different number of unknown parameter 
Figure 7 compares the deduced value with the target value for various number of observation data $(=65,33,11)$ without adding a priori condition. This figure shows that the deduced value is unstable if the number of observation data is insufficient $(=11)$ while the drag coefficient deduced by the inversion method is accurate if the number of the observation data is sufficient $(=65,33)$ compared with the number of the unknown parameter $(=25)$. These results leads that it is important to chose an appropriate number of unknown parameters by considering the number of the observation data.
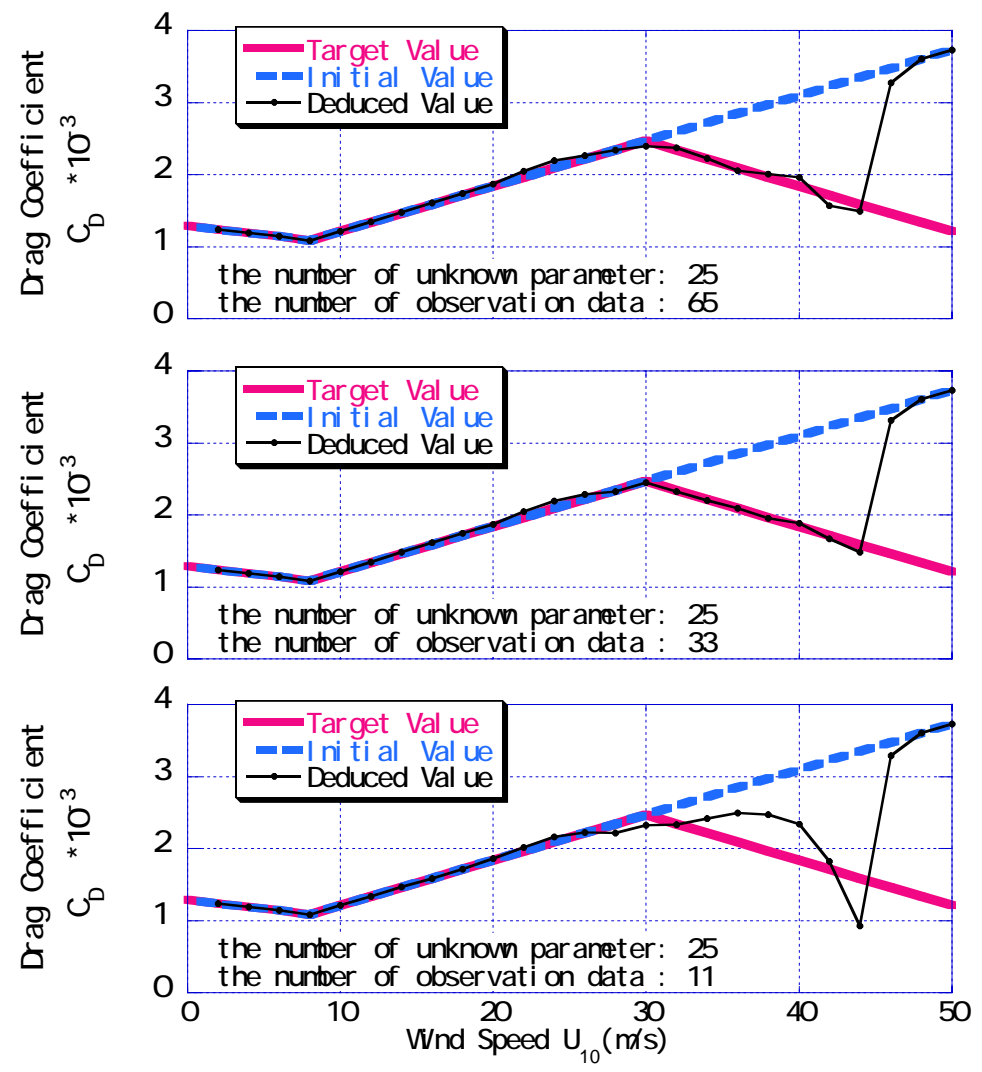

Figure 7. Deduced Drag coefficients with different number of observation data

The panels in the left column on Figure 8 compare the deduced value with the target value, where the computations are carried out for different weighting coefficients $\left(W=0,10^{3}, 10^{4}, 10^{5}\right)$ with the a priori condition of $x_{n}-x_{n-1} \approx 0$. Although the deduced value is unstable when $W=0$, no addition of the a priori condition, the deduced value approaches the target value by adding the a priori condition. The deduced value is almost equal to the target value for $W=10^{4}$. These results lead that the accuracy of the inversion can be improved by adding the a priori condition to the cost function even if the number of the wave observation data is insufficient and the deduced value is unstable. The deduced value of the wind speed around $30 \mathrm{~m} / \mathrm{s}$ is parted from the target value for $W=10^{5}$ compared with the one with $W=10^{4}$. It is suggested that the weighting coefficient for the a priori condition is too large compared with the observation error.

The panels in the right column in Figure 8 compares the deduced value with the target value, where the computations are carried out for the same condition as left column respectively with a different type of the a priori condition of $x_{n+1}-2 x_{n}+x_{n-1} \approx 0$ from the left column. As well as the left panels, the deduced value was accurately corrected to the target value from the initial value for $W=10^{4}$. The deduced value in high wind speed is little unstable compared with the left figures especially in the case of $W=10^{5}$. 

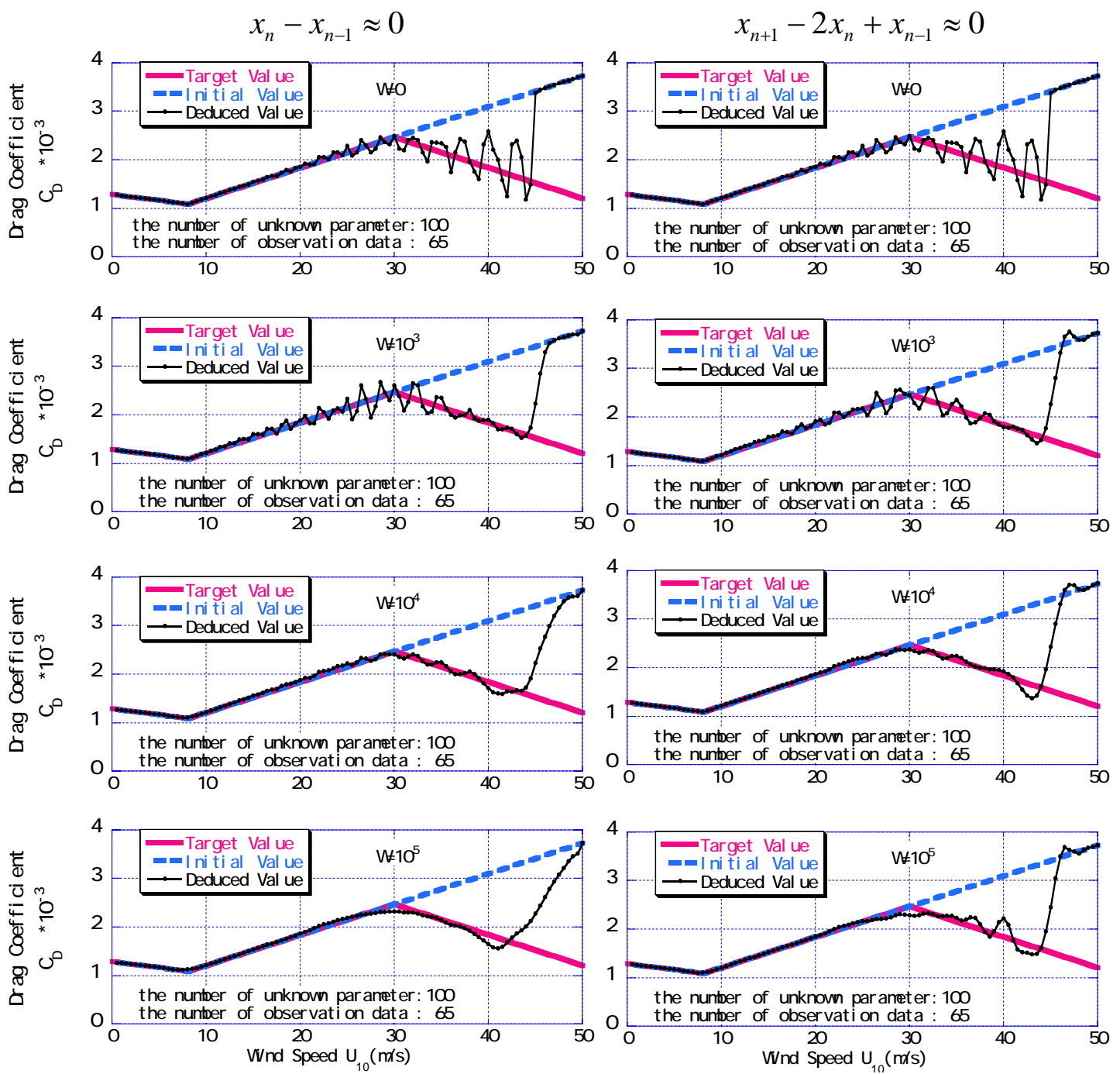

Figure 8. Deduced drag coefficients with different weighting coefficient of the a priori conditions

In the above experiments, the behaviour of the parameter in a wind speed higher than $30 \mathrm{~m} / \mathrm{s}$ was examined by assuming the initial value given by Mitsuyasu \& Honda's equation. In the following examinations, in order to examine the possibility of the inverse estimation from the arbitrary initial value, the initial value was changed and assumed as constant value given by $1 * 10^{-3}$ whose parameter value differs from the target value in almost all the range of wind speed as seen in Fig. 9. Figure 9 compares the deduced value with the target value, where the computations are carried out for different weighting coefficients $\left(W=0,10^{3}, 10^{4}, 10^{5}\right)$ with the a priori condition of $x_{n}-x_{n-1} \approx 0$. As seen in the figures, the deduced value approaches the target value by adding the a priori condition although the deduced value around the wind speed of $30 \mathrm{~m} / \mathrm{s}$ was also unstable when $W=0$ due to the large difference between the initial value and the target value is large. Therefore, it was confirmed that the new model can estimate optimal parameters appropriately by adding a priori condition with adequate weight coefficient $W$ even if the initial value is arbitrary. 

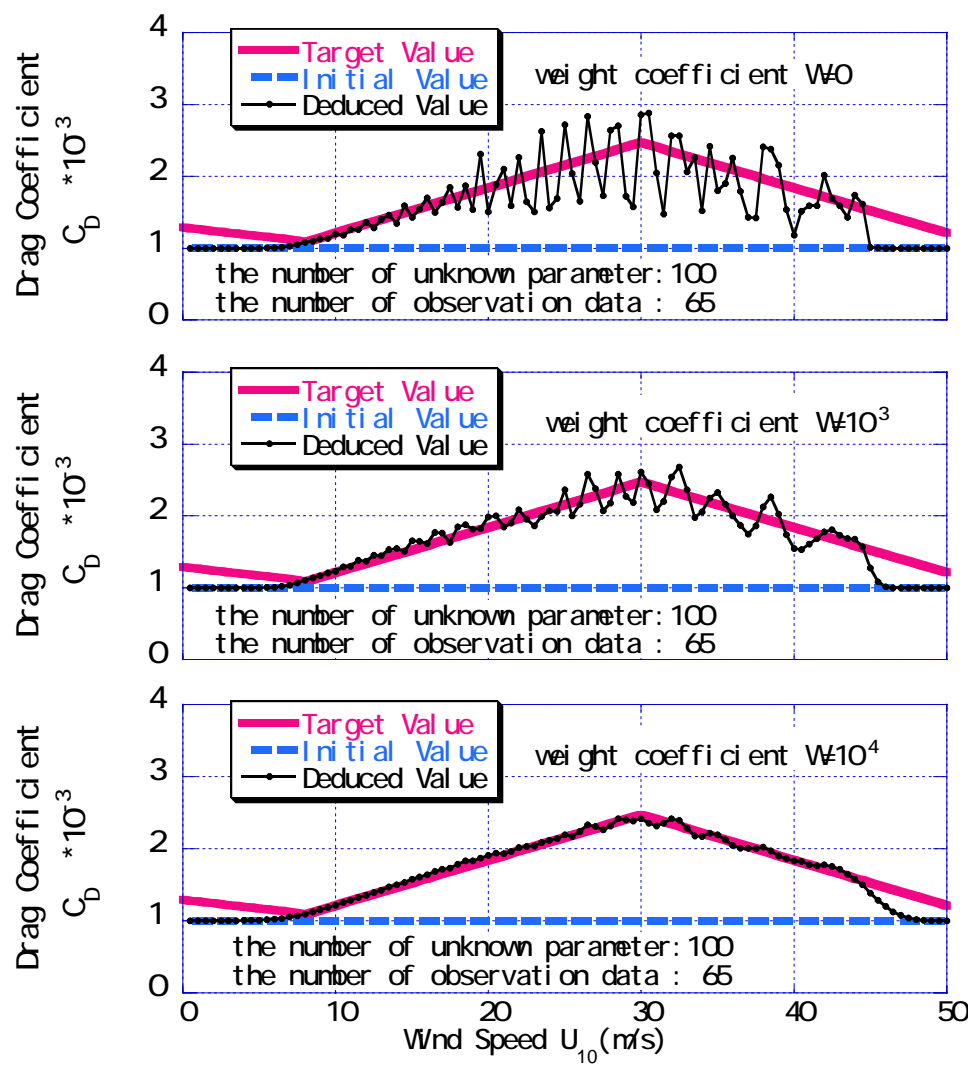

Figure 9. Deduced drag coefficient computed with constant initial value by $1^{\star} 10^{-3}$

\section{RESULTS}

For the clarification of the mechanism on energy transfer from high wind to waves, the ADWAM, a wave prediction model incorporating data assimilation method, was modified to deduce the sea surface drag coefficients as its control variables. Then, the validity of the new model was verified through identical twin experiments in deep sea conditions. Also, the behavior of the deduced parameter was investigated for several experiments. The major conclusions are as follows.

1) The new model can estimate the optimal parameters appropriately from arbitrary assumed initial value by adding a priori condition with adequate weight coefficient even if the target values are unpredictable.

2) The drag coefficient deduced by the proposed inversion method is accurate if the number of the observation data is sufficient compared with that of unknown parameters.

3) The accuracy in the inversion of the drag coefficient can be improved by adding a priori condition even if the number of the observation data is insufficient.

\section{FUTURE PLAN}

1) The wave prediction accuracy in severe sea conditions may be improved if the drag coefficients are clarified by applying the method to the actual wave data measured under severe sea conditions. We will therefore try to apply the proposed method to the actual wave observation data.

2) Furthermore we will improve this model so as to be applied to the time series data of directional wave spectrum as the observation data instead of those of the significant wave height that was used as the observation data in this study. This improvement will enable the method more accurate and reliable.

\section{ACKNOWLEDGMENTS}

This work was supported by Grant-in-Aid for Scientific Research (No. 20360222) from the Japan Society for Promotion of Science (JSPS). 


\section{REFERENCES}

Andreas E. L. : An algorithm to predict the turbulent air-sea fluxes in high-wind, spray conditions, 12th Conf. on interaction of sea and atmosphere, CD-ROM, 3.4.

Giering, R. 1995. The Adjoint Model Compiler, MPI report.

Hersbach, H. (1998) : Application of the adjoint of the WAM model to inverse wave modeling, J. Geophys. Res. Vol.103,(C5), pp.10469-10487.

Powell, M.D., Vickery, P.J and T.A. Reinhold (2003): Reduced drag coefficient for high wind speeds in tropical cyclones, Nature, 422, pp.279-283

Hashimoto, $\mathrm{N}$ and K. Matsuura,Evaluation of cost function in wave data assimilation with WAM Proc. 6th Int. Conf. Hydrodynamics VI, Theory and applications,pp.199-204.,2004.01.

Hashimoto, N., K. Matsuura, T. Nagai, H. Kawai. 2007. Application of Adjoint WAM to NOWPHAS Wave Observation Network for Establishment of Reasonable Observation Network, Proc. 30th Int. Conf. Coastal Eng. 'Coastal Engineering 2006',Vol.1, 565-577.

Hashimoto, N., K. Matsuura, H. Kawai, Attempt for Accuracy Improvement of Wave Hindcasting / Forecasting in Coastal Sea and Inner Sea by Data Assimilation, Proc. 31th Int. Conf. Coastal Eng. 'Coastal Engineering 2008',2009.05

Hersbach H. and Janssen P.A.E.M. 1999. Improvement of the Short Fetch Behavior in the WAM model, J. Atmos. Ocean. Techn., 16, pp.884-892.

Holthuijsen, L. H. et at. 2000. SWAN Cycle III version 40.11, USER MANUAL, Delft University of Technology, 124p.

Hsiao, S. V. and Shemdin, O.H. 1983. Measurements of wind velocity and pressure with a wave follower during MARSEN, J. Geophys. Res., 88(C14), 9841-9849.

Janssen P. E. A. M. 1991. Quasi-linear theory of wind-wave generation applied to wave forecasting, J. Physical Oceanography, 21, 1631-1642.

Kobayashi T., Adachi T. and Yasuda T. 2003. Estimation of Wave Fields with Open Boundaries by Ap-plying an Adjoint Model, Asian Pacific Coasts 2003, 8p.

Komen, G. J. et. al. 1994. Dynamics and Modelling of Ocean Waves. Cambridge University Press, 532p. 1994.

Mitsuyasu, H. and T. Honda, 1982. Wind-induced growth of water waves, J. Fluid Mech., 123, 425442.

NOAA/NWS/NCEP/OMB technical note 166, 110p.

Tolmann, H. L.1999. User manual and system documentation of WAVEWATCH-III Version 1.18,

The WAMDI Group (13 Authors). 1988. The WAM model-A third generation ocean wave prediction model, J. Phys. Oceanogr., 18, pp.1378-1391.

Zhang, W., W. Perrie. and W. Li, 2006. Impacts of Waves and Sea Spray on Midlatitude Storm Structure and Intensity, Monthly Weather Review, 2006 American Meteorological Society, Vol. 134, 2418-2442. 